\title{
Distinct Regions of Prefrontal Cortex Mediate Resistance and Vulnerability to Depression
}

\author{
Michael Koenigs, ${ }^{1}$ Edward D. Huey, ${ }^{2}$ Matthew Calamia, ${ }^{3}$ Vanessa Raymont, ${ }^{2,4}$ Daniel Tranel, ${ }^{3}$ and Jordan Grafman ${ }^{2}$ \\ ${ }^{1}$ Department of Psychiatry, University of Wisconsin-Madison, Madison, Wisconsin 53719, ${ }^{2}$ Cognitive Neuroscience Section, National Institute of \\ Neurological Disorders and Stroke-National Institutes of Health, Bethesda, Maryland 20892-1440, ${ }^{3}$ Department of Neurology, University of Iowa Hospitals and \\ Clinics, Iowa City, Iowa 52242, and ${ }^{4}$ Vietnam Head Injury Study, Henry M. Jackson Foundation, National Naval Medical Center, Bethesda, Maryland 20889
}

The neuroanatomical correlates of depression remain unclear. Functional imaging data have associated depression with abnormal patterns of activity in prefrontal cortex (PFC), including the ventromedial ( $v m P F C)$ and dorsolateral (dlPFC) sectors. If vmPFC and dlPFC are critical neural substrates for the pathogenesis of depression, then damage to either area should affect the expression of depressive symptoms. Using patients with brain lesions we show that, relative to nonfrontal lesions, bilateral vmPFC lesions are associated with markedly low levels of depression, whereas bilateral dorsal PFC lesions (involving dorsomedial and dorsolateral areas in both hemispheres) are associated with substantially higher levels of depression. These findings demonstrate that vmPFC and dorsal PFC are critically and causally involved in depression, although with very different roles: vmPFC damage confers resistance to depression, whereas dorsal PFC damage confers vulnerability.

Key words: depression; emotion; prefrontal cortex; ventromedial; dorsolateral; neuropathology

\section{Introduction}

A primary aim in the neuroscientific study of affective disorders is to identify the responsible dysfunctional brain circuits. In particular, the role of prefrontal areas in depression has been a major focus of previous work (Drevets, 1998, 2007; Davidson et al., 2002). Perhaps the most widely accepted division of prefrontal cortex, based on anatomical connectivity and functional specialization, is between the dorsolateral and ventromedial sectors (Rosenkilde, 1979; Kringelbach and Rolls, 2004; Zald, 2007). Neuroimaging studies of resting state activity associate depression with abnormally high levels of ventromedial prefrontal cortex (vmPFC) activity (Drevets et al., 1992; Biver et al., 1994; Nofzinger et al., 2005; Greicius et al., 2007), but abnormally low levels of dorsolateral prefrontal cortex (dlPFC) activity (Baxter et al., 1989; Bench et al., 1993; Biver et al., 1994; Galynker et al., 1998; Mayberg et al., 1999). These resting state data suggest that the pathogenesis of depression may depend on relative hyperactivity in vmPFC and hypoactivity in dlPFC (Mayberg, 1997), although more recent studies using cognitive activation paradigms reveal a more complex profile of PFC activity (e.g., Wagner et al., 2006). Regardless, imaging data alone cannot determine whether the abnormal activity in either vmPFC or dlPFC reflects the underlying cause of the disorder or a secondary effect of the disorder. The question of causality, however, can be addressed through the

\footnotetext{
Received May 22, 2008; revised 0ct. 13, 2008; accepted 0ct. 14, 2008.

This work was supported in part by the National Institute of Neurological Disorders and Stroke (NINDS) intramural research program, DAMD17-01-1-0675 (J.G.), National Institute on Drug Abuse Grant R01 DA022549 (D.T.), and NINDS Grant P01 NS19632 (D.T.).

Correspondence should be addressed to Dr. Jordan Grafman, National Institutes of Health, 10 Center Drive, MSC 1440, Bethesda, MD 20892-1440. E-mail: grafmanj@ninds.nih.gov.

DOI:10.1523/JNEUROSCI.2324-08.2008

Copyright $\odot 2008$ Society for Neuroscience $\quad$ 0270-6474/08/2812341-08\$15.00/0
}

study of humans with focal brain lesions. If vmPFC hyperactivity and dIPFC hypoactivity are indeed causally involved in the pathogenesis of depression, then damage to either area would presumably affect the development of depression, but with opposite effects. More specifically, vmPFC lesions would confer resistance to depression, whereas dIPFC lesions would confer vulnerability to depression.

To test this prediction, we assessed the severity of depressive symptoms in individuals with focal lesions involving either vmPFC or dorsal PFC. Previous studies investigating the effect of discrete brain lesions on depression have yielded mixed results with respect to lesion location and depression severity. Initial studies in stroke patients reported an association between left anterior lesions and poststroke depression (Robinson et al., 1984; Parikh et al., 1988), but multiple subsequent studies failed to replicate this finding (for review, see Singh et al., 1998; Carson et al., 2000; Yu et al., 2004) and meta-analyses of poststroke depression studies reveal no clear association between lesion location and depression (Carson et al., 2000; Yu et al., 2004). The lack of a consistent relationship between lesion location and depression in these studies could be due factors such as the following: (1) nearly all patients in these studies suffered unilateral damage, so the contralesional hemisphere could potentially provide some compensation of function, (2) in some studies patients were assessed only in the acute recovery phase, so in these cases the reported effects may not endure in the chronic epoch, and (3) none of these studies differentiated between ventromedial and dorsolateral PFC lesions. Here, we report the first study examining the effects of chronic, focal, bilateral lesions of vmPFC and dorsal PFC on depression severity. 

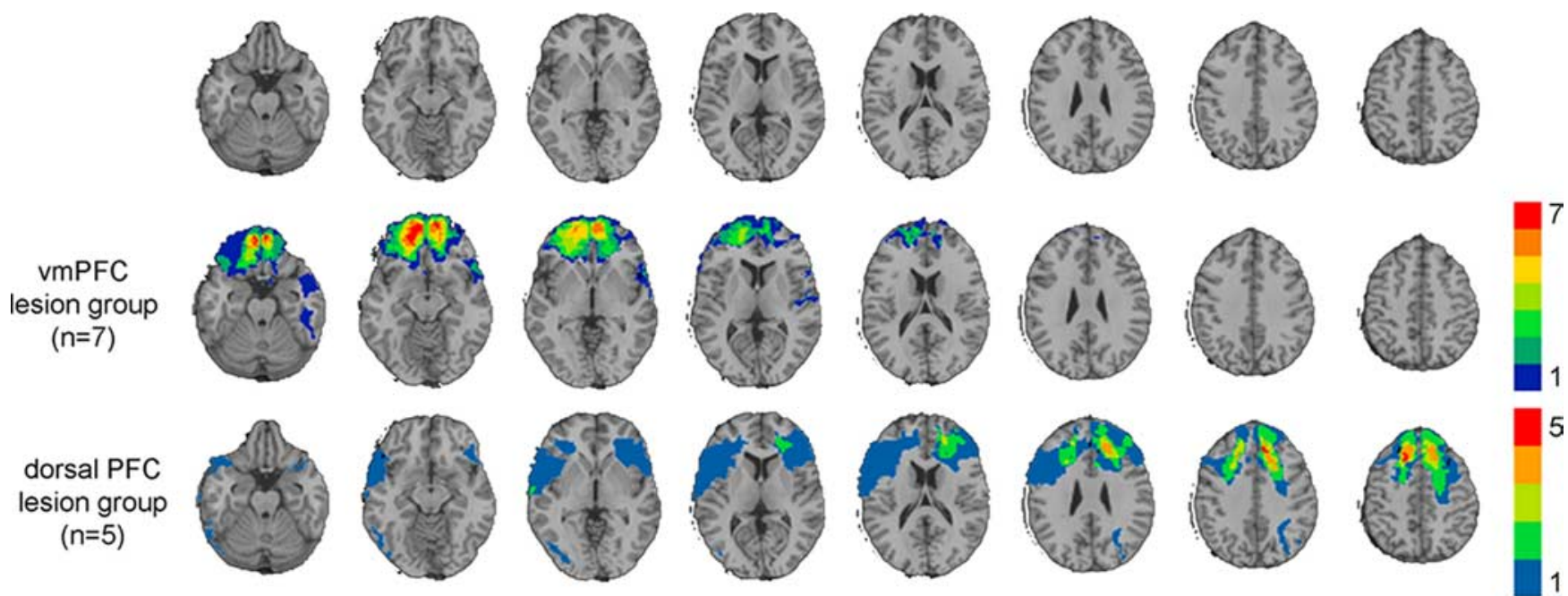

Figure 1. Lesion overlaps of VHIS prefrontal lesion groups. Color indicates the number of overlapping lesions at each voxel. Top row, Transverse slices of a normal healthy brain, for reference. From left to right, $z=-22,-12,-2,8,18,28,38$, and 48 . In each slice, the right hemisphere is on the reader's left. Second row, Lesion overlap for the vmPFC lesion group. Third row, Lesion overlap for the dorsal PFC lesion group.

\section{Materials and Methods}

Patient registries. We studied two separate samples of patients with focal brain lesions. One sample is the Vietnam Head Injury Study (VHIS) registry, which is comprised of American veterans who suffered brain damage mainly from penetrating head injuries in the Vietnam War. The other sample is the Patient Registry of the Cognitive Neuroscience Division at the University of Iowa, which is comprised largely of Iowans who suffered brain damage from ischemic stroke, subarachnoid hemorrhage, or neurosurgical resection. Because the two patient samples differ with respect to gender, lesion onset, and experience of emotional trauma, convergent findings from these two samples would elucidate in compelling manner the relative roles of PFC subregions in depression.

VHIS participants. We drew one set of participants from the W.F. Caveness VHIS registry, which originally included 1221 American soldiers who survived penetrating brain wounds suffered in Vietnam. The VHIS has been organized in three phases. Phase 1 was the initial enrollment, which occurred between 1967 and 1970. For Phase 2 , the 1118 veterans still alive were invited to participate in an extensive follow-up clinical study at Walter Reed Army Medical Center between August 1981 and August 1984. Of the 1118 survivors, 520 participated in the Phase 2 study. Comparison subjects $(n=85)$ were recruited from VA files of non-head-injured soldiers who had served in Vietnam the same years and were within the same age range as soldiers on the Caveness registry. One-hundred and ninety-nine head-injured and 54 non-head-injured subjects from Phase 2 participated in Phase 3, which included a psychiatric evaluation by a neuropsychiatrist (Vanessa Raymont). We conducted Phase 3 between April 2003 and November 2006 at Bethesda National Naval Medical Center. Preinjury characteristics of the participants were available from military and VA records. All subjects gave informed written consent before completing the study.

VHIS lesion analysis. We acquired CT data during the Phase 3 testing period. We acquired Axial CT scans without contrast at Bethesda Naval Hospital on a GE Medical Systems Light Speed Plus CT scanner in helical mode. We reconstructed the images with an in-plane voxel size of 0.4 $\mathrm{mm} \times 0.4 \mathrm{~mm}$, overlapping slice thickness of $2.5 \mathrm{~mm}$ and a $1 \mathrm{~mm}$ slice interval. We determined lesion location and volume from CT images using the Analysis of Brain Lesion (ABLe) software (Makale et al., 2002; Solomon et al., 2007) contained in MEDx v3.44 (Medical Numerics) with enhancements to support the Automated Anatomical Labeling (AAL) atlas (Tzourio-Mazoyer et al., 2002). We defined lesions by manual tracing in all relevant slices of the CT image. A trained neuropsychiatrist (Vanessa Raymont) performed the tracings, which were then reviewed by an observer (Jordan Grafman) who was blind to the results of the clinical evaluations. The CT image of each brain was spatially nor- malized to a CT template brain image in MNI space (Collins et al., 1994). Individuals' normalized scans were used for the computation of group overlaps. We defined two regions of interest (ROIs): the vmPFC and the dlPFC. The vmPFC ROI was defined as those areas of PFC inferior to $\mathrm{z}=$ 0 and medial to $\mathrm{x}=20$ and $\mathrm{x}=-20$. These criteria outlined an area comprising the ventral portion of the medial prefrontal cortex (below the level of the genu of the corpus callosum) and medial portion of the orbital surface (approximately the medial one-third of the orbitofrontal cortex in each hemisphere) as well as the subjacent white matter. The dlPFC ROI was defined as those areas of PFC superior to $\mathrm{z}=0$ and lateral to $\mathrm{x}=$ -10 and $\mathrm{x}=10$. A patient was included in the vmPFC group if his lesion occupied vmPFC in both hemispheres, but did not occupy any portion of dlPFC in either hemisphere. A patient was included in the dorsal PFC group if his lesion occupied dlPFC in both hemispheres, but did not occupy any portion of vmPFC in either hemisphere. The selection of bilateral lesions precluded the possibility that normal function could be sustained by the intact contralesional hemisphere.

VHIS patient group characteristics. Based on the aforementioned neuroanatomical criteria, we identified seven patients with damage to vmPFC in both hemispheres, but no damage to dlPFC in either hemisphere ("vmPFC lesion group") (Fig. 1), and we identified five patients with damage to dlPFC in both hemispheres, but no damage to vmPFC in either hemisphere ("dorsal PFC lesion group") (Fig. 1). Because damage to dlPFC in both hemispheres was always accompanied by damage to the intervening cortex (dorsomedial PFC), we refer to this group as the "dorsal PFC lesion group." In addition, we studied veterans with lesions not involving PFC ("non-PFC lesion group"; $n=101$ ) and veterans with no brain damage ("non-brain-damaged group"; $n=54$ ). Among the nonPFC lesion group, damage primarily affected the lateral aspect of cerebral cortex. Lesions in this group were distributed throughout temporal, parietal, and occipital cortex, but largely spared deep structures such as basal ganglia, insula, and thalamus. Groups were well matched for age, education, basic cognitive function, and preservice cognitive function (Table 1). Lesion patient groups were well matched for cognitive decline after injury (Table 1). Lesion volume for the vmPFC group (mean 40.1 $\mathrm{cm}^{3}$, SD 19.6) was not significantly different from lesion volume for the dorsal PFC group (mean $62.0 \mathrm{~cm}^{3}$, SD 22.3) $(t=-1.8 ; p=0.10)$.

VHIS depression assessment. We assessed the VHIS participants with three independent measures of depression: (1) the Beck Depression Inventory, Second Edition (BDI-II) (Beck et al., 1996) (2) the Structured Clinical Interview for DSM-IV-TR Axis I disorders, Non-Patient edition (SCID-N/P) (First et al., 2002), and (3) the Neurobehavioral Rating Scale (NRS) (Levin et al., 1987). The BDI-II is a 21-item self-report instrument for measuring the severity of specific symptoms of depression. Partici- 
Table 1. VHIS patient group characteristics

\begin{tabular}{|c|c|c|c|c|c|c|c|}
\hline Group (lesion location) & $n$ & Age & Sex (\% male) & Education (years) & MMSE & Preservice AFQT (percentile) & AFQT change \\
\hline vmPFC & 7 & $58.7(4.1)$ & 100 & $14.1(3.4)$ & $28.7(1.0)$ & $55.9(24.8)$ & $-5.8(12.9)$ \\
\hline Dorsal PFC & 5 & $57.6(2.3)$ & 100 & $13.6(3.0)$ & $28.3(1.5)$ & $46.6(30.7)$ & $-3.6(10.3)$ \\
\hline Non-PFC & 101 & $58.5(3.1)$ & 100 & $14.9(2.5)$ & $28.7(1.8)$ & $61.9(25.8)$ & $-7.6(18.8)$ \\
\hline Non-brain damaged & 54 & $59.0(3.4)$ & 100 & $15.2(2.5)$ & $29.1(1.3)$ & $65.4(22.9)$ & $3.9(14.5)$ \\
\hline
\end{tabular}

For "Age," "Education," "MMSE," "Preservice AFQT (percentile)," and "AFQT Change," mean values are given with standard deviations in parentheses. "Age" refers to years at the time of BDI-II administration. "Sex (\% male)" is the percentage of male subjects. "MMSE" is the Mini Mental State Examination (Folstein et al., 1975), a test of basic cognitive function administered at the time of the BDI-II; "AFQT" (Armed Forces Qualification Test) is a measure of basic cognitive function; "Preservice AFQT (percentile)" is the AFQT percentile at the time of enlistment; "AFQT Change" is the difference between AFQT score at the time of enlistment and the BDI-II. For brain-injured veterans, "AFQT Change" is a measure of cognitive decline following injury.

Table 2. lowa patient group characteristics

\begin{tabular}{lllllr}
\hline Group (lesion location) & $n$ & $\begin{array}{l}\text { Age at } \\
\text { onset }\end{array}$ & Sex (\% male) & Education (years) & FSIQ \\
\hline vmPFC & 13 & $49.0(13.3)$ & 54 & $13.1(2.4)$ & $105.4(15.4)$ \\
Nonfrontal & 238 & $48.2(14.2)$ & 46 & $12.9(2.9)$ & $99.3(13.7)$ \\
\hline
\end{tabular}

For"Age at onset," "Education," and"FSIQ," mean values are given with standard deviations in parentheses. "Age at onset" refers to years at the time of lesion onset. "Sex (\% male)" is the percentage of male subjects. "FSIQ" refers to WAIS-III Full Scale IQ, a measure of postlesion cognitive function. FSIQ is based on available data from 119 patients in the nonfrontal group and 11 patients in the vmPFC group. Groups did not significantly differ with respect to any of the variables.

pants rate each item on a scale of $0-3$, with greater numbers indicating greater severity. An overall BDI score $(0-63)$ is derived by summing the severity ratings for each item. In the BDI-II manual, Beck et al. (1996) report the mean BDI-II scores for groups of individuals with different levels of depression severity, as determined by clinical evaluation. "Nondepressed" individuals had mean BDI-II scores of 7.7 (SD 5.9), whereas "mildly depressed," "moderately depressed," and "severely depressed" individuals had mean BDI-II scores of 19.1 (SD 5.7), 27.4 (SD 10.0), and 33.0 (SD 12.0), respectively. Thus, for our study, we interpreted any BDI-II score of 8 or below ( $>2$ SD below the mean for "mildly depressed" individuals) as a negligible level of depression ("low" depression severity), and any score of 20 or above as clinically significant depression ("high" depression severity). Scores 9 through 19 were considered "intermediate" depression severity.

The SCID-N/P is a structured interview used for making DSM-IV Axis I diagnoses. A psychiatrist (Vanessa Raymont) used the SCID-N/P to evaluate each participant for major psychiatric disorders.

The NRS is a 27-item instrument for measuring the severity of behavioral sequelae of head injury. One item pertains specifically to depressed mood. The rater assesses the severity of depressive mood from 1 (not present) to 7 (extremely severe) based on the spontaneous behavior of the patient (i.e., the rater did not question the patient specifically about depressed mood). For this study the NRS was completed by research assistants trained in neuropsychological assessment. The research assistants completed the NRS after interacting with the participant for $\sim 25 \mathrm{~h}$ over a $5 \mathrm{~d}$ period.

Iowa participants. We drew a second set of participants from the $\mathrm{Pa}$ tient Registry of the Division of Cognitive Neuroscience at the University of Iowa. All participants in the group analysis had focal, stable lesions that could be clearly identified on MR or CT scans, and they were free of dementia, substance abuse, and premorbid psychiatric disorder. All participants were capable of comprehending the test instructions and cooperating with the experiment. Neuropsychological and neuroanatomical studies were all conducted in the chronic phase of recovery (more than 3 months after lesion onset). All participants gave informed consent before completing the study.

Iowa lesion analysis. We determined lesion location from CT or MR images. We initially screened the Iowa Patient Registry for individuals with bilateral vmPFC lesions by selecting those patients with lesions occupying F11 and/or F12 in both hemispheres, according to the Damasio and Damasio (1989) templates. From these patients we selected patients with bilateral damage to same areas described for the VHIS patients: the ventral portion of the medial prefrontal cortex (below the level of the genu of the corpus callosum) and medial portion of the orbital surface (approximately the medial one-third of the orbitofrontal cortex in each hemisphere) as well as the subjacent white matter. Patients were excluded if their lesions extended to the lateral surface in region F07 (the dlPFC) or significantly outside of PFC. Lesion etiologies for the vmPFC group were tumor resection $(n=8)$ and anterior communicating artery aneurysm rupture/clipping $(n=5)$. Two of the vmPFC patients had lesions that extended out of PFC; one patient's lesion included right superior anterior temporal lobe, and another patient's lesion included right caudate. The nonfrontal comparison group was comprised of those individuals who did not have any damage to frontal areas (F01-F14) and who had completed either the Beck Depression Inventory, First Edition Amended (BDI-IA) (Beck and Steer, 1993) or the BDI-II. Lesion etiologies for the nonfrontal group were primarily ischemic stroke, hemorrhage, or surgical resection.

Iowa patient characteristics. In the Iowa neurological registry, we identified 13 patients with damage to vmPFC in both hemispheres, but no significant damage to dlPFC in either hemisphere ("Iowa vmPFC lesion group"). There were no patients in the Iowa registry with focal lesions involving dlPFC in both hemispheres. For comparison purposes, we identified those patients with lesions not involving frontal cortex ("Iowa nonfrontal lesion group"; $n=238$ ). Lesions in the nonfrontal group included lateral areas of temporal, parietal, and occipital cortex (e.g., MCA strokes), temporal pole (e.g., temporal lobectomies), medial occipital cortex (e.g., PCA strokes), as well as basal ganglia and thalamus (e.g., hemorrhage). The two groups did not significantly differ with respect to sex, age of lesion onset, education, or basic cognitive function after brain injury (Table 2).

Iowa depression assessment. We assessed depression with either the BDI-IA or the BDI-II. The two versions are similar but not identical: between the two versions (both of which have 21 items), there are 17 conserved items but 4 different items. In the vmPFC group, 6 patients completed the BDI-IA and 7 completed the BDI-II. In the nonfrontal comparison group, 118 patients completed the BDI-IA and 120 completed the BDI-II. For overall BDI scores, we converted BDI-IA scores to BDI-II scores according to the conversion chart published in BDI-II manual (Beck et al., 1996). We used the same criteria as in the VHIS study to classify overall scores as indicating "low" $(0-8)$, "intermediate" (9$19)$, or "high" (20-63) levels of depression.

\section{Results}

\section{VHIS patient depression data}

To test our hypothesis that vmPFC damage would reduce depression severity whereas dorsal PFC damage would increase depression severity, we used Fisher's exact test to determine if the proportions of individuals with "low" or "high" levels of depression severity differed among VHIS patient groups (Fig. 2). The proportion of individuals with "low" depression severity in the vmPFC group (7 of 7) was significantly greater than in the dorsal PFC lesion group ( 1 of $5 ; p=0.005$ ), non-PFC lesion group ( 65 of $101 ; p=0.05$ ), and non-brain-damaged group (24 of $54 ; p=$ 0.006). In contrast, the proportion of individuals with "high" depression severity in the dorsal PFC group (3 of 5) was significantly greater than in the vmPFC lesion group ( 0 of $7 ; p=0.045$ ) and non-PFC lesion group ( 10 of $101 ; p=0.012$ ), and marginally greater than the non-brain-damaged group (13 of $54 ; p=0.10$ ). 


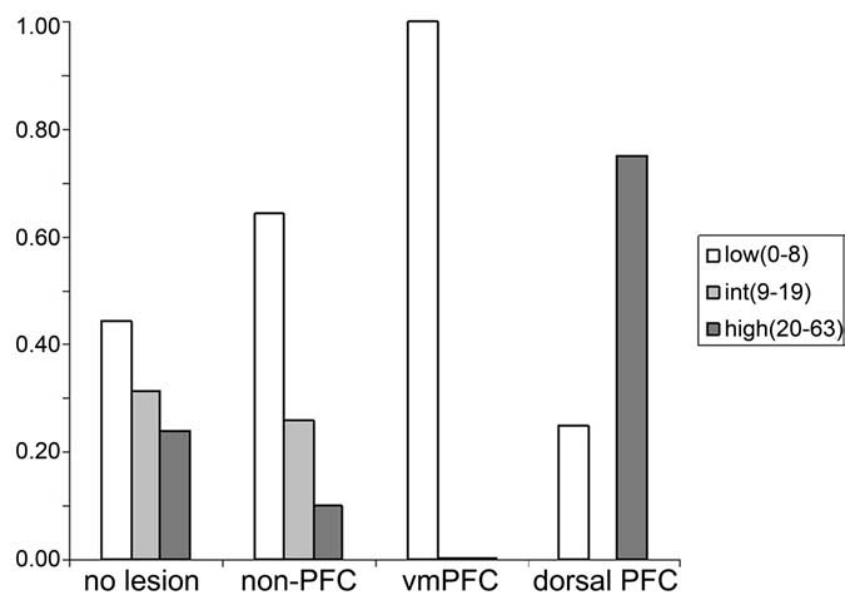

Figure 2. VHIS BDI-II scores. $y$-Values indicate the proportion of individuals who were classified as "low" (white bars), "intermediate" (light gray bars), or "high" (dark gray bars) depression severity for each group. Patient groups are denoted on the $x$-axis. "No lesion" refers to the non-brain-damaged group. The vmPFC lesion group had the significantly greatest proportion of "low" severity BDI-II scores, whereas the dorsal PFC lesion group had the significantly greatest proportion of "high" severity BDI-II scores.

These data indicate that bilateral vmPFC damage was associated with relatively low depression severity, whereas bilateral dorsal PFC damage was associated with relatively high depression severity.

As a complementary analysis, we performed a parametric analysis of the BDI-II scores, and found that mean depression scores differed significantly among groups $(F=3.3 ; p=0.021)$. The specific pattern of results was identical to the nonparametric analysis: the mean BDI-II score of the vmPFC lesion group (4.4) was significantly lower than the mean BDI-II score of both the non-PFC lesion group $(8.9 ; t=-3.3 ; p=0.004)$ and non-braindamaged group (11.6; $t=-4.3 ; p<0.001)$, whereas the mean BDI-II score of the dorsal PFC lesion group (19.0) was significantly greater than the mean BDI-II score of the non-PFC lesion group $(t=2.3 ; p=0.021)$, and marginally greater than the nonbrain-damaged group $(t=1.6 ; p=0.12)$. The parametric analysis thus confirms the differential effects of vmPFC and dorsal PFC lesions on depression.

One possible reason for the between-group differences in BDI-II scores could be a difference in the prevalence of posttraumatic stress disorder (PTSD) at the time of the BDI-II assessment. PTSD is commonly associated with symptoms of depression (Regier et al., 1998; Devane et al., 2005), and vmPFC damage is associated with a reduction in PTSD among combat veterans (Koenigs et al., 2008). Thus, the absence of higher BDI-II scores in the vmPFC group could be attributable to the absence of PTSD in this group. Indeed, none of the seven vmPFC patients were diagnosed with concurrent PTSD at the time of BDI administration, whereas $13 / 101$ in the non-PFC lesion group, 1 of 5 in the dorsal PFC lesion group, and 15 of 54 in the non-brain-damaged group were diagnosed with concurrent PTSD at the time of the BDI administration. After removal of these PTSD-positive individuals, Fisher's exact test showed that the proportion of "low" depression severity individuals in the vmPFC group remained significantly greater than the dorsal PFC lesion group ( $p=0.02)$ and non-brain-damaged group $(p=0.04)$, and marginally greater than the non-PFC lesion group $(p=0.09)$. These data indicate that the absence of PTSD did not fully account for the abnormally low BDI-II scores in the vmPFC group.
Because depression is a disorder involving multiple characteristic symptoms, which may be experienced in varying degrees, the observed overall reduction in BDI-II scores among the vmPFC patients and overall elevation in BDI-II scores among the dorsal PFC patients raise an important follow-up question: does vmPFC or dorsal PFC damage affect all depressive symptoms to a similar extent, or are the differences in overall BDI-II score driven by a subset of symptoms? To answer this question, we compared the groups' mean severity ratings for each of the 21 items of the BDI-II (Fig. 3). Multiple studies report a two-factor structure for the BDI-II: one factor of "cognitive/affective" items (e.g., sadness, worthlessness, self-dislike) and another factor of "somatic" items (e.g., changes in appetite, tiredness, concentration difficulty) (Beck et al., 1996; Steer et al.,1999; Whisman et al., 2000; Storch et al., 2004) (see also Buckley et al., 2001; Vanheule et al., 2008). The 14 items receiving the lowest self-reported severity among vmPFC patients were exactly the same 14 items that loaded onto the original "cognitive/affective" factor reported in BDI-II manual (Beck et al., 1996). There was a significant difference among groups for mean ratings of the "cognitive/affective" items $(F=$ $2.8 ; p=0.04)$. For these items, the vmPFC lesion group had a significantly lower mean score (0.07) than either the non-PFC lesion group $(0.35 ; t=-4.5 ; p<0.001)$ or the non-braindamaged group $(0.48 ; t=-5.1 ; p<0.001)$. The vmPFC lesion group did not have significantly lower scores for the remaining (primarily "somatic") items $(F=1.7 ; p=0.17)$. This analysis indicates that bilateral vmPFC damage selectively diminishes the cognitive/affective symptoms of depression.

As a second independent measure of depression in the VHIS patients, a psychiatrist assessed whether or not each participant met the DSM-IV criteria for "lifetime" and/or "current" major depressive disorder (MDD) using the SCID-N/P. The SCID-N/P includes "lifetime" and "current" diagnoses for other mood disorders including bipolar affective disorder (I and II), dysthymic disorder, and depressive disorder not-otherwise-specified (DDNOS). The "lifetime" diagnosis pertained to any point in the participant's life, including preinjury service in Vietnam. As such, the "lifetime" diagnosis is not necessarily informative with respect to the effect of brain injury on depression. In contrast, a diagnosis of "current" MDD unambiguously indicates the presence of symptoms after the injury. The SCID data for mood disorders are presented in Table 3. The proportion of individuals meeting DSM-IV criteria for "lifetime" MDD was not significantly different between groups (Fisher's exact test; all $p$ values $>$ $0.16)$, nor were the proportions of individuals meeting DSM-IV criteria for other "current" mood disorders (Fisher's exact test; all $p$ values $>0.21)$. However, the proportion of individuals meeting DSM-IV criteria for "current" MDD was significantly greater for the dorsal PFC lesion group ( 2 of 5 ) than for the non-PFC lesion group ( 1 of $101 ; p=0.005$ ) or non-brain-damaged group ( 0 of $54 ; p=0.006)$. Thus, bilateral dorsal PFC lesions were associated with a relatively high prevalence of subsequent major depression.

As a third measure of depression, each VHIS patient was assessed with the NRS, which includes one item pertaining specifically to depressed mood. There were no significant differences in the rating of depression severity on the NRS among the five groups $(F=0.43 ; p=0.79)$. Although the between-group differences were not significant, the pattern of depression severity among groups was consistent with the BDI-II and SCID-N/P data: the mean rating of depression severity for the vmPFC lesion group (1.17) was lower than the non-PFC lesion group (1.41), non-brain-damaged group (1.37), and dorsal/ventral PFC lesion 


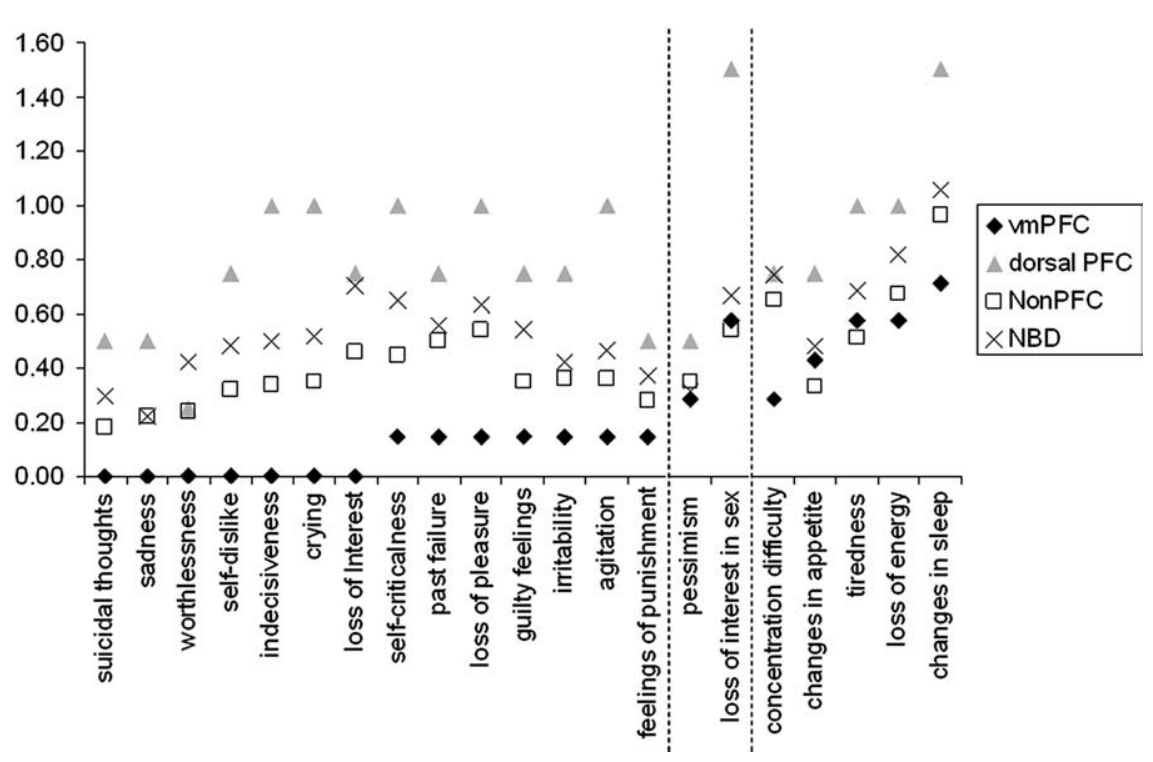

Figure 3. Mean severity scores for individual BDI-Il items. The 14 items to the left of both dashed lines are "cognitive/affective" symptoms, whereas the 5 items to the right of both dashed lines are the "somatic" symptoms. The 2 items between the dashed lines did not load on either factor.

Table 3. SCID-N/P assessment of Axis I depressive disorders

\begin{tabular}{llllll}
\hline Group (lesion location) & Current bipolar & Current dysthymia & Current DD-NOS & Lifetime MDD & Current MDD \\
\hline $\operatorname{vmPFC~}(n=7)$ & 0.00 & 0.14 & 0.00 & 0.43 & 0.00 \\
Dorsal PFC $(n=5)$ & 0.00 & 0.00 & 0.00 & 0.40 & $\mathbf{0 . 4 0}$ \\
Non-PFC $(n=101)$ & 0.00 & 0.03 & 0.01 & 0.20 & 0.01 \\
Non-brain damaged $(n=54)$ & 0.00 & 0.09 & 0.04 & 0.26 & 0.00 \\
\hline
\end{tabular}

Values are proportions of individuals in each group receiving the diagnosis. "DD-NOS," Depressive disorder not-otherwise specified;"MDD," major depressive disorder. Statistically significant proportions for each disorder (based on Fisher's exact test) are in bold.

group (1.33), whereas the mean rating of depression severity for the dorsal PFC lesion group was higher (1.80).

\section{Relationship of depression and cognitive or motor impairment}

One possible explanation of the relatively high depression severity among veterans with lesions involving dorsal PFC is that their symptoms of depression could be a consequence of significant impairment in cognitive and/or motor function. To address this possibility, we used a multiple regression analysis to determine whether BDI-II score among all brain-injured VHIS patients was significantly predicted by neuropsychological measures of cognitive function or neurological measures of motor function. We included the following measures in the analysis: MMSE, a gross measure of intellectual function; AFQT change, a measure of overall cognitive decline; Boston Naming Test, a measure of object naming and aphasia; Token Test, a measure of language comprehension; Verbal Fluency, a measure of executive function; and the presence/absence of ataxia, paresis, and voluntary movement abnormality. The model was not significant $\left(F_{(8,147)}=1.5, p=\right.$ 0.18 ), and explained only $2.3 \%$ of the variance in BDI-II score (adjusted $R^{2}=0.023$ ), indicating that none of the measures of cognitive or motor function were strong predictors of BDI-II score in the VHIS patient sample. These results suggest that the elevated depression among veterans with dorsal PFC damage is not simply an affective response to cognitive or motor impairment.

\section{Iowa patient depression data}

Given the considerable effect of PFC lesion location on depressive symptomotology among veterans in the VHIS, we sought to de- termine whether the same pattern would be observed in a different population of patients with PFC lesions. The proportion of individuals with "low" depression severity in the Iowa vmPFC group (12 of 13) was significantly greater than in the Iowa nonfrontal comparison group (109 of 238; $p=0.001$ ) (Fig. 4). Again we confirmed this finding with a parametric test: the mean BDI score of the Iowa vmPFC lesion group (4.7) was significantly lower than the mean BDI score of the Iowa nonfrontal lesion comparison group $(10.3 ; t=-5.3$; $p<0.001)$. These results mirror the VHIS data; focal bilateral vmPFC damage was associated with relatively low depression severity.

Again, we determined whether bilateral vmPFC damage was associated with a reduction in particular symptoms of depression (supplemental Table 1, available at www.jneurosci.org as supplemental material). The 13 bilateral vmPFC patients from the Iowa registry uniformly endorsed the complete absence of three symptoms: sadness, loss of interest, and feelings of punishment, all of which load onto the "cognitive/affective" factor. The Iowa vmPFC patient group's highest ratings of symptom severity were for change in appetite and tiredness, which load onto the "somatic" factor, and pessimism, which loads onto neither factor. Thus, like the VHIS patients with focal bilateral vmPFC lesions, the Iowa vmPFC patients exhibit less severe "cognitive/affective" symptoms than "somatic" symptoms of depression.

In addition, we identified one patient in the Iowa registry who represents an intriguing case of an apparent alleviation of severe depression after a bilateral vmPFC lesion. Although no records of formal psychiatric assessments were available, per secondary report the patient was being treated for depression when she attempted suicide 11 years ago by means of a gunshot to the head. The gunshot destroyed most of ventral PFC, including vmPFC bilaterally, but left intact most of dorsal PFC (supplemental Fig. 1 , available at www.jneurosci.org as supplemental material). The patient's neuropsychologist, neurosurgeon, and long-term boyfriend all remarked that her depression was markedly diminished after the brain injury (boyfriend, speaking 16 months after the injury: "no sign of depression whatsoever since the accident"; neuropsychologist: "she never shows distress, worry, or anger"). Her postinjury self-report data, collected at the University of Iowa 16 months after the injury, corroborate this impression. On the BDI-IA, the patient had a total score of 11 . The specific items she endorsed were severe loss of libido; moderate levels of fatigability and indecisiveness, and mild levels of sense of failure, selfaccusations, irritability, and weight loss. She endorsed the complete absence of 14 items, including sadness, self-dislike, guilt, and suicidal ideation. Despite the lack of data from formal preinjury and postinjury psychiatric assessments, which precludes any definitive conclusions regarding the effect of the lesion on her mood state, the available data for this case are consistent with vmPFC playing a causal role in the experience of "cognitive/affective" symptoms of depression. 


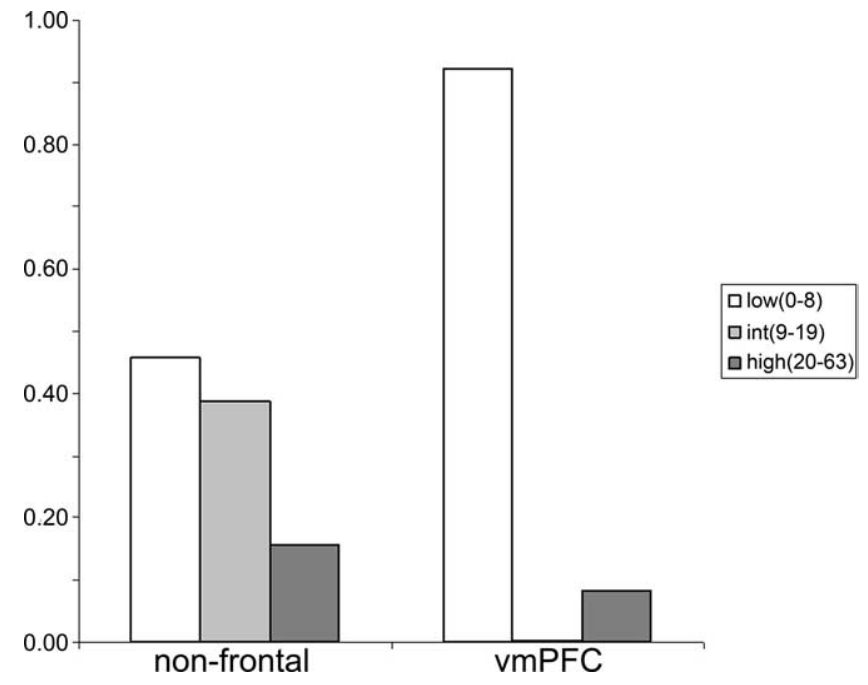

Figure 4. Iowa BDI scores. $y$-Values indicate the proportion of individuals who were classified as "low" (white bars), "intermediate" (light gray bars), or "high" (dark gray bars) depression severity for each group. Patient groups are denoted on the $x$-axis. The vmPFC lesion group had a significantly greater proportion of "low" severity BDI scores than the nonfrontal lesion group.

\section{Discussion}

In sum, we found opposite effects of vmPFC and dorsal PFC damage on depression. In the VHIS patient sample, veterans with bilateral vmPFC damage reported significantly lower depression severity than did veterans with damage involving other areas of the brain or veterans with no brain damage, particularly for the cognitive/affective symptoms of depression. This finding was replicated in the Iowa sample: neurological patients with bilateral vmPFC damage reported significantly lower depression severity than did patients with damage involving posterior areas of the brain. In addition, one patient with a premorbid history of depression experienced a dramatic alleviation of depressive symptoms, particularly the cognitive/affective symptoms, after bilateral vmPFC damage. Conversely, VHIS patients with bilateral dorsal PFC damage reported significantly greater depression severity than veterans with damage involving other areas of the brain and were more frequently diagnosed with current major depression than were veterans with damage involving other areas of the brain or veterans with no brain damage. VHIS patients with unilateral dIPFC damage also reported relatively high levels of depression severity (supplemental results; supplemental Fig. 2, available at www.jneurosci.org as supplemental material). These data support the prediction that vmPFC lesions would confer resistance to depression whereas dlPFC lesions would confer susceptibility to depression, and suggest that vmPFC and dIPFC are causally involved in the experience of depression.

The possible mechanisms for the differential effects of vmPFC and dorsal PFC lesions on depression warrant discussion. First we consider the finding of diminished depression among vmPFC patients. Previous lesion studies associate vmPFC damage with a marked lack of self-insight (Barrash et al., 2000; Beer et al., 2006), as well as a reduction in certain types of negative affect, particularly shame, guilt, embarrassment, and regret (Camille et al., 2004; Beer et al., 2006; Koenigs et al., 2007). One common feature of these emotions is that they all involve an element of selfawareness or self-reflection. We speculate that the observed lack of depression among vmPFC patients could be attributable to an impairment of self-insight or self-reflection. Data from specific
BDI items support this interpretation: vmPFC patients reported conspicuously low levels of "cognitive/affective" symptoms (such as guilt, self-dislike, and sadness) but normal levels of "somatic" symptoms (such as fatigue and changes in sleeping or appetite). Because the "cognitive/affective" items ostensibly involve a greater degree of self-reflection and rumination than the "somatic" items, one can conceive of the vmPFC patients' lack of depression as a secondary effect of a primary lack of selfawareness and self-reflection. This interpretation holds that vmPFC is critical for self-insight and thus also critical for the self-reflective negative affect that is fundamental to depression; a loss of this capacity of self-insight/self-reflection after vmPFC damage may reduce many symptoms of depression.

Impairment in self-insight/self-reflection could also theoretically account for our previous finding of diminished PTSD among veterans with vmPFC lesions (Koenigs et al., 2008), as PTSD is characterized by the distressful experience of negative affect (e.g., anxiety, guilt) associated with the rumination of past events. The finding of both diminished depression and diminished PTSD among patients with vmPFC lesions supports the assertion that mood and anxiety disorders share a common higher order dimension of general distress or "negative affectivity" (Clark and Watson, 1991; Watson, 2005), and moreover, that vmPFC may be a critical neural substrate for this common dimension, perhaps by virtue of its role in self-insight. An interesting follow-up study would be to determine whether vmPFC patients exhibit certain objective (behavioral or physiological) correlates of depression or anxiety while reporting no subjective experience of depressed or anxious mood.

With respect to the elevated depression severity among VHIS patients with dorsal PFC damage, we believe our results are consistent with recent functional imaging data demonstrating the recruitment of dlPFC during the regulation of negative emotion through reappraisal/suppression strategies (Ochsner et al., 2002, 2004; Lévesque et al., 2003; Phan et al., 2005; Eippert et al., 2007). If the reappraisal/suppression of negative affect is a protective mechanism against depression, and if dlPFC is necessary for this function, then one would expect elevated depression among individuals with dlPFC damage. We believe that a defect in the regulation of negative affect attributable to dlPFC damage is indeed a plausible mechanism for the observed elevation in depression in the dorsal PFC lesion group.

However, it is important to point out limitations on the conclusions that can be made with respect to the role of dlPFC in depression, based on the results of this study. For one, the patients did not have "pure" bilateral dIPFC lesions. Since the intervening cortex (the dorsomedial PFC) was also damaged in each of the dorsal PFC patients, the contribution of dorsomedial PFC damage to the observed results cannot be discounted. Second, although both parametric and nonparametric analyses of $\mathrm{BDI}$ scores indicate greater depression in the dorsal PFC lesion patients than in the non-PFC lesion patients, the difference in BDI scores between the dorsal PFC lesion patients and the nonbrain-damaged comparison subjects did not reach significance. However, the proportion of dorsal PFC lesion patients diagnosed with current MDD was significantly greater than that of either comparison group, including the non-brain-damaged subjects. Finally, all patients in the dorsal PFC lesion group were adult males, so the findings may not generalize to all cases of depression. Given these limitations, we regard our conclusions about the causal role of dIPFC in depression as preliminary, pending replication in additional patient samples.

One unique feature of this study is the sample sizes of the 
target groups: 20 cases of focal bilateral vmPFC damage (seven in the VHIS registry, 13 in the Iowa registry) and five cases of bilateral dlPFC damage. The 20 total patients with focal, bilateral vmPFC damage is, to our knowledge, the largest such group ever reported in a single study. And to our knowledge, ours is also the first study to assemble a group of patients with focal lesions involving dlPFC in both hemispheres. Moreover, the relatively large sample sizes of key comparison groups (101 VHIS patients with non-PFC lesions, 54 veterans with no brain damage, and 238 Iowa patients with nonfrontal damage) provide a stable point of reference and allow for meaningful statistical analyses. Nonetheless, it will be important to corroborate our observed pattern of results with additional cases of prefrontal damage.

A second unique feature of this study is the use of two vmPFC patient samples with distinct characteristics. Whereas all VHIS patients are men of similar age who experienced emotionally traumatic events and lesion onset in early adulthood, the Iowa patients are both men and women with various ages of lesion onset who did not systematically experience emotional trauma before their brain injuries. The fact that nearly identical results were obtained in both samples of vmPFC patients supports the generalizability of the findings, and allows us to rule out a host of attribute variables as likely explanations for the observed results.

It is noteworthy that the case study presented in this study (the woman who evinced a dramatic alleviation of depressive symptoms after a failed suicide attempt that inflicted bilateral vmPFC damage) bears remarkable resemblance to a previously reported case (Ellenbogen et al., 2005). In this case, a 33-year-old man attempted suicide with a cross-bow, inflicting severe damage within vmPFC. Afterward he was "indifferent to his situation and inappropriately cheerful." Together, these two extraordinary cases underscore the critical and causal role that vmPFC plays in mediating negative affect.

Furthermore, the findings of this study accord with previous studies of neuroanatomically specific treatments for mood and anxiety disorders, such as psychosurgery and exogenous brain stimulation. Subcaudate tractotomy, a surgical procedure that interrupts white matter tracts connecting the vmPFC to subcortical structures, has shown efficacy in reducing symptoms of depression and anxiety (Cosgrove, 2000; Shields et al., 2008). And as would be predicted by the results of our study, vegetative symptoms of depression (e.g., changes in appetite, sleep, and energy) were less likely to improve with subcaudate tractotomy than depressed mood or anxiety (Hodgkiss et al., 1995). Similarly, a study of deep-brain stimulation reports antidepressant effects of inhibiting subgenual PFC white matter (Mayberg et al., 2005), which is adjacent to and interconnected with the vmPFC area described in our study. In addition, studies of transcranial magnetic stimulation have reported antidepressant effects of stimulating dlPFC (Berman et al., 2000; Herwig et al., 2003; Avery et al., 2006). Our results support the notion that treatments aimed at selectively inhibiting vmPFC and/or stimulating dlPFC could have efficacy in alleviating depression.

\section{References}

Avery DH, Holtzheimer PE 3rd, Fawaz W, Russo J, Neumaier J, Dunner DL, Haynor DR, Claypoole KH, Wajdik C, Roy-Byrne P (2006) A controlled study of repetitive transcranial magnetic stimulation in medicationresistant major depression. Biol Psychiatry 59:187-194.

Barrash J, Tranel D, Anderson SW (2000) Acquired personality disturbances associated with bilateral damage to the ventromedial prefrontal region. Dev Neuropsychol 18:355-381.

Baxter LR Jr, Schwartz JM, Phelps ME, Mazziotta JC, Guze BH, Selin CE, Gerner RH, Sumida RM (1989) Reduction of prefrontal cortex glucose metabolism common to three types of depression. Arch Gen Psychiatry 46:243-250.

Beck AT, Steer RA (1993) Manual for the beck depression inventory. San Antonio: Psychological Corporation.

Beck AT, Steer RA, Brown, GK (1996) Manual for the beck depression inventory-II. San Antonio: Psychological Corporation.

Beer JS, John OP, Scabini D, Knight RT (2006) Orbitofrontal cortex and social behavior: integrating self-monitoring and emotion-cognition interactions. J Cogn Neurosci 18:871-879.

Bench CJ, Friston KJ, Brown RG, Frackowiak RS, Dolan RJ (1993) Regional cerebral blood flow in depression measured by positron emission tomography: the relationship with clinical dimensions. Psychol Med 23:579-590.

Berman RM, Narasimhan M, Sanacora G, Miano AP, Hoffman RE, Hu XS, Charney DS, Boutros NN (2000) A randomized clinical trial of repetitive transcranial magnetic stimulation in the treatment of major depression. Biol Psychiatry 47:332-337.

Biver F, Goldman S, Delvenne V, Luxen A, De Maertelaer V, Hubain P, Mendlewicz J, Lotstra F (1994) Frontal and parietal metabolic disturbances in unipolar depression. Biol Psychiatry 36:381-388.

Buckley TC, Parker JD, Heggie J (2001) A psychometric evaluation of the BDI-II in treatment-seeking substance abusers. J Subst Abuse Treat 20:197-204.

Camille N, Coricelli G, Sallet J, Pradat-Diehl P, Duhamel JR, Sirigu A (2004) The involvement of the orbitofrontal cortex in the experience of regret. Science 304:1167-1170.

Carson AJ, MacHale S, Allen K, Lawrie SM, Dennis M, House A, Sharpe M (2000) Depression after stroke and lesion location: a systematic review. Lancet 356:122-126.

Clark LA, Watson D (1991) Tripartite model of anxiety and depression: psychometric evidence and taxonomic implications. J Abnorm Psychol 100:316-336.

Collins DL, Neelin P, Peters TM, Evans AC (1994) Automatic 3D intersubject registration of MR volumetric data in standardized Talairach space. J Comput Assist Tomogr 18:192-205.

Cosgrove GR (2000) Surgery for psychiatric disorders. CNS Spectr 5:43-52. Damasio H, Damasio AR (1989) Lesion analysis in neurospychology. New York: Oxford UP.

Davidson RJ, Pizzagalli D, Nitschke JB, Putnam K (2002) Depression: perspectives from affective neuroscience. Annu Rev Psychol 53:545-574.

Devane CL, Chiao E, Franklin M, Kruep EJ (2005) Anxiety disorders in the 21 st century: status, challenges, opportunities, and comorbidity with depression. Am J Manag Care 11:S344-353.

Drevets WC (1998) Functional neuroimaging studies of depression: the anatomy of melancholia. Annu Rev Med 49:341-361.

Drevets WC (2007) Orbitofrontal cortex function and structure in depression. Ann N Y Acad Sci 1121:499-527.

Drevets WC, Videen TO, Price JL, Preskorn SH, Carmichael ST, Raichle ME (1992) A functional anatomical study of unipolar depression. J Neurosci 12:3628-3641.

Eippert F, Veit R, Weiskopf N, Erb M, Birbaumer N, Anders S (2007) Regulation of emotional responses elicited by threat-related stimuli. Hum Brain Mapp 28:409-423.

Ellenbogen JM, Hurford MO, Liebeskind DS, Neimark GB, Weiss D (2005) Ventromedial frontal lobe trauma. Neurology 64:757.

First MB, Spitzer RL, Gibbon M, Williams JBW (2002) Structured clinical interview for DSM-IV-TR axis I disorders, research version, patient edition. (SCID-I/P) New York: Biometrics Research, New York State Psychiatric Institute.

Folstein MF, Folstein SE, McHugh PR (1975) "Mini-mental state". A practical method for grading the cognitive state of patients for the clinician. J Psychiatr Res 12:189-198.

Galynker II, Cai J, Ongseng F, Finestone H, Dutta E, Serseni D (1998) Hypofrontality and negative symptoms in major depressive disorder. J Nucl Med 39:608-612.

Greicius MD, Flores BH, Menon V, Glover GH, Solvason HB, Kenna H, Reiss AL, Schatzberg AF (2007) Resting-state functional connectivity in major depression: abnormally increased contributions from subgenual cingulate cortex and thalamus. Biol Psychiatry 62:429-437.

Herwig U, Lampe Y, Juengling FD, Wunderlich A, Walter H, Spitzer M, Schönfeldt-Lecuona C (2003) Add-on rTMS for treatment of depres- 
sion: a pilot study using stereotaxic coil-navigation according to PET data. J Psychiatr Res 37:267-275.

Hodgkiss AD, Malizia AL, Bartlett JR, Bridges PK (1995) Outcome after the psychosurgical operation of stereotactic subcaudate tractotomy, 19791991. J Neuropsychiatry Clin Neurosci 7:230-234.

Koenigs M, Young L, Adolphs R, Tranel D, Cushman F, Hauser M, Damasio A (2007) Damage to the prefrontal cortex increases utilitarian moral judgements. Nature 446:908-911.

Koenigs M, Huey ED, Raymont V, Cheon B, Solomon J, Wassermann EM, Grafman J (2008) Focal brain damage protects against post-traumatic stress disorder in combat veterans. Nat Neurosci 11:232-237.

Kringelbach ML, Rolls ET (2004) The functional neuroanatomy of the human orbitofrontal cortex: evidence from neuroimaging and neuropsychology. Prog Neurobiol 72:341-372.

Lévesque J, Eugène F, Joanette Y, Paquette V, Mensour B, Beaudoin G, Leroux JM, Bourgouin P, Beauregard M (2003) Neural circuitry underlying voluntary suppression of sadness. Biol Psychiatry 53:502-510.

Levin HS, High WM, Goethe KE, Sisson RA, Overall JE, Rhoades HM, Eisenberg HM, Kalisky Z, Gary HE (1987) The neurobehavioural rating scale: assessment of the behavioural sequelae of head injury by the clinician. J Neurol Neurosurg Psychiatry 50:183-193.

Makale M, Solomon J, Patronas NJ, Danek A, Butman JA, Grafman J (2002) Quantification of brain lesions using interactive automated software. Behav Res Methods Instrum Comput 34:6-18.

Mayberg HS (1997) Limbic-cortical dysregulation: a proposed model of depression. J Neuropsychiatry Clin Neurosci 9:471-481.

Mayberg HS, Liotti M, Brannan SK, McGinnis S, Mahurin RK, Jerabek PA, Silva JA, Tekell JL, Martin CC, Lancaster JL, Fox PT (1999) Reciprocal limbic-cortical function and negative mood: converging PET findings in depression and normal sadness. Am J Psychiatry 156:675-682.

Mayberg HS, Lozano AM, Voon V, McNeely HE, Seminowicz D, Hamani C, Schwalb JM, Kennedy SH (2005) Deep brain stimulation for treatmentresistant depression. Neuron 45:651-660.

Nofzinger EA, Buysse DJ, Germain A, Price JC, Meltzer CC, Miewald JM, Kupfer DJ (2005) Alterations in regional cerebral glucose metabolism across waking and non-rapid eye movement sleep in depression. Arch Gen Psychiatry 62:387-396.

Ochsner KN, Bunge SA, Gross JJ, Gabrieli JD (2002) Rethinking feelings: an FMRI study of the cognitive regulation of emotion. J Cogn Neurosci 14:1215-1229.

Ochsner KN, Ray RD, Cooper JC, Robertson ER, Chopra S, Gabrieli JD, Gross JJ (2004) For better or for worse: neural systems supporting the cognitive down- and up-regulation of negative emotion. Neuroimage 23:483-499.

Parikh RM, Lipsey JR, Robinson RG, Price TR (1988) A two year longitudinal study of poststroke mood disorders: prognostic factors related to one and two year outcome. Int J Psychiatry Med 18:45-56.
Phan KL, Fitzgerald DA, Nathan PJ, Moore GJ, Uhde TW, Tancer ME (2005) Neural substrates for voluntary suppression of negative affect: a functional magnetic resonance imaging study. Biol Psychiatry 57:210-219.

Regier DA, Rae DS, Narrow WE, Kaelber CT, Schatzberg AF (1998) Prevalence of anxiety disorders and their comorbidity with mood and addictive disorders. Br J Psychiatry Suppl:24-28.

Robinson RG, Kubos KL, Starr LB, Rao K, Price TR (1984) Mood disorders in stroke patients. Importance of location of lesion. Brain 107:81-93.

Rosenkilde CE (1979) Functional heterogeneity of the prefrontal cortex in the monkey: a review. Behav Neural Biol 25:301-345.

Shields DC, Asaad W, Eskandar EN, Jain FA, Cosgrove GR, Flaherty AW, Cassem EH, Price BH, Rauch SL, Dougherty DD (2008) Prospective assessment of stereotactic ablative surgery for intractable major depression. Biol Psychiatry 64:449-454.

Singh A, Herrmann N, Black SE (1998) The importance of lesion location in poststroke depression: a critical review. Can J Psychiatry 43:921-927.

Solomon J, Raymont V, Braun A, Butman JA, Grafman J (2007) Userfriendly software for the analysis of brain lesions (ABLe). Comput Methods Programs Biomed 86:245-254

Steer RA, Ball R, Ranieri WF, Beck AT (1999) Dimensions of the Beck Depression Inventory-II in clinically depressed outpatients. J Clin Psychol 55:117-128.

Storch EA, Roberti JW, Roth DA (2004) Factor structure, concurrent validity, and internal consistency of the Beck Depression Inventory-Second Edition in a sample of college students. Depress Anxiety 19:187-189.

Tzourio-Mazoyer N, Landeau B, Papathanassiou D, Crivello F, Etard O, Delcroix N, Mazoyer B, Joliot M (2002) Automated anatomical labeling of activations in SPM using a macroscopic anatomical parcellation of the MNI MRI single-subject brain. Neuroimage 15:273-289.

Vanheule S, Desmet M, Groenvynck H, Rosseel Y, Fontaine J (2008) The factor structure of the Beck Depression Inventory-II: an evaluation. Assessment 15:177-187.

Wagner G, Sinsel E, Sobanski T, Köhler S, Marinou V, Mentzel HJ, Sauer H, Schlösser RG (2006) Cortical inefficiency in patients with unipolar depression: an event-related FMRI study with the Stroop task. Biol Psychiatry 59:958-965.

Watson D (2005) Rethinking the mood and anxiety disorders: a quantitative hierarchical model for DSM-V. J Abnorm Psychol 114:522-536.

Whisman MA, Perez JE, Ramel W (2000) Factor structure of the Beck Depression Inventory-Second Edition (BDI-II) in a student sample. J Clin Psychol 56:545-551.

Yu L, Liu CK, Chen JW, Wang SY, Wu YH, Yu SH (2004) Relationship between post-stroke depression and lesion location: a meta-analysis. Kaohsiung J Med Sci 20:372-380.

Zald DH (2007) Orbital versus dorsolateral prefrontal cortex: anatomical insights into content versus process differentiation models of the prefrontal cortex. Ann N Y Acad Sci 1121:395-406. 